
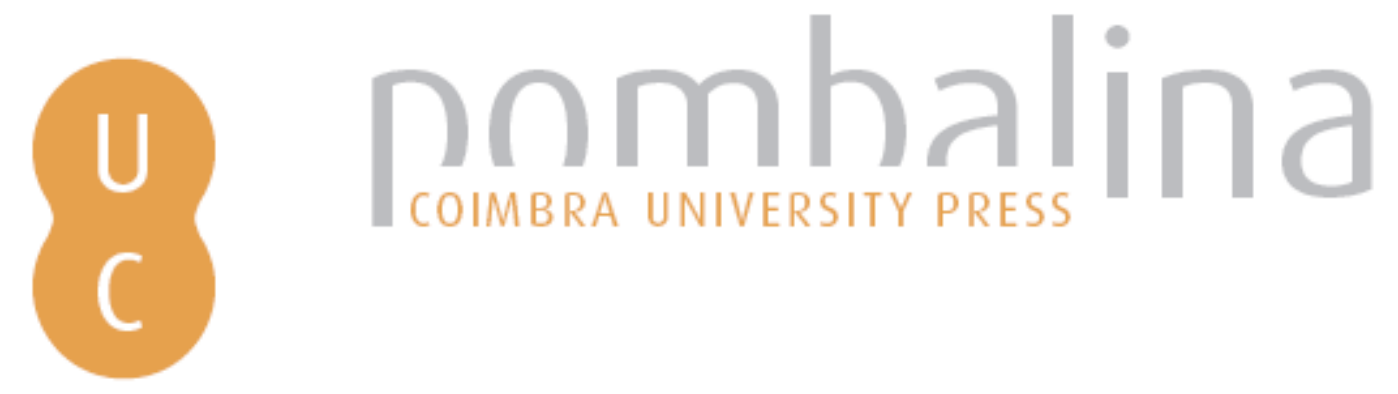

\title{
Aplicação de ferramentas de análise e estatística espacial: retrato do sector estratégico do comércio na cidade de Lisboa
}

Autor(es): $\quad$ Dias, Pedro; Rocha, Jorge; Tenedório, José António; Tomé, Teresa

Publicado por: Imprensa da Universidade de Coimbra

URL

persistente: URI:http://hdl.handle.net/10316.2/37076

DOI: $\quad$ DOI:http://dx.doi.org/10.14195/978-989-26-0983-6_23

Accessed : $\quad$ 26-Apr-2023 11:34:49

A navegação consulta e descarregamento dos títulos inseridos nas Bibliotecas Digitais UC Digitalis, UC Pombalina e UC Impactum, pressupõem a aceitação plena e sem reservas dos Termos e Condições de Uso destas Bibliotecas Digitais, disponíveis em https://digitalis.uc.pt/pt-pt/termos.

Conforme exposto nos referidos Termos e Condições de Uso, o descarregamento de títulos de acesso restrito requer uma licença válida de autorização devendo o utilizador aceder ao(s) documento(s) a partir de um endereço de IP da instituição detentora da supramencionada licença.

Ao utilizador é apenas permitido o descarregamento para uso pessoal, pelo que o emprego do(s) título(s) descarregado(s) para outro fim, designadamente comercial, carece de autorização do respetivo autor ou editor da obra.

Na medida em que todas as obras da UC Digitalis se encontram protegidas pelo Código do Direito de Autor e Direitos Conexos e demais legislação aplicável, toda a cópia, parcial ou total, deste documento, nos casos em que é legalmente admitida, deverá conter ou fazer-se acompanhar por este aviso.

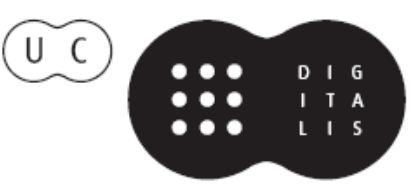




\section{$\forall$ \\ TAS DAS I JORNADAS LUSÓFONAS DE CIÊNCIAS E TECNOLOGIAS DE INFORMAÇÃO GEOGRÁFICA}

Editores

José Gomes dos Santos

Cidália Fonte

Rui Ferreira de Figueiredo

Alberto Cardoso

Gil Gonçalves

José Paulo Almeida

Sara Baptista 


\title{
ARTIGO 23
}

\section{APLICAÇÃo de FERRAMENTAS DE ANÁlise e ESTATística ESPACIAL - RETRATO DO SECTOR ESTRATÉGICO DO COMÉRCIO NA CIDAdE DE LISBOA}

\author{
DIAS, Pedro ${ }^{1}$; ROCHA, Jorge ${ }^{2}$; TENEDÓRIO, José António 3 \& TOMÉ, Teresa ${ }^{4}$ \\ 1 e 3 e-GEO, FCSH-UNL, Avenida de Berna, 26-C, 1069- 061 Lisboa, Portugal. Tel: (+351) 2179083 00; \\ pedro.dias@fcsh.unl.pt - ja.tenedorio@fcsh.unl.pt \\ 2 CEG, IGOT-UL, Alameda da Universidade, 1600-214 Lisboa, Portugal; Tel: (+351) 217940218 ; jorge. \\ rocha@campus.ul.pt \\ ${ }^{4}$ DMEI, Câmara Municipal de Lisboa, Campo Grande, $25-7^{\circ} \mathrm{C}, 1749-099$ Lisboa, Portugal. Tel: $(+351)$ \\ 217988 357; ana.teresa.tome@cm-lisboa.pt
}

\section{RESUMO}

Neste artigo são apresentados métodos aplicados ao sector estratégico do co-mércio na cidade de Lisboa de forma a visualizar qual o retracto deste sector na cidade. Para tal são utilizadas ferramentas específicas de análise espacial e de estatística espacial.

No primeiro grupo são usadas as ferramentas "Kernel Density", "Minus" e "Plus" e no se-gundo as ferramentas "Kriging" e "Hot Spot Analysis". A informação utilizada é composta pelo recenseamento comercial dos "estabelecimentos de comércio de retalho" e dos "es-tabelecimentos de restauração e bebidas", dos anos 1995, 2000 e 2009.

\section{PALAVRAS-CHAVE}

SIG, Análise espacial, Estatística espacial, Sectores estratégicos, Comércio. 


\title{
PRACTICAL APPLICATION OF SPATIAL STATISTICS \\ ANALYSIS TOOLS: THE COMMERCE STRATEGIC \\ SECTOR STATUS OF THE CITY OF LISBON
}

\begin{abstract}
In this paper are presented methods applied to trade strategic sector in Lisbon city in order to see which picture of this sector in the city. For this purpose are used spe-cific tools of spatial and spatial statistics analysis. In the first group are used the tools "Kernel Density", "Minus" and "Plus" and the second the tools "Kriging" and "Hot Spot Analysis". The information used is composed by the trade census of "retailing establish-ments" and "restaurants and drinks establishments", of the years 1995, 2000 and 2009.
\end{abstract}

\section{KEYWORDS}

GIS, Spatial analysis, Spatial statistics analysis, Strategic sectors, Trade.

\section{INTRODUÇÃO}

Neste artigo são apresentadas os métodos de análises espaciais e estatísticas espaciais aplicadas ao sector estratégico do comércio na cidade de Lisboa, de forma a visualizar qual o retracto deste sector na cidade. É de salientar que o sector divide-se em dois ní-veis, os estabelecimentos de comércio de retalho (retalho) e os estabelecimentos de res-tauração e bebidas (restauração). São usadas ferramentas e métodos de geoprocessa-mento das extensões Spatial Analyst Tools e Spatial Statistics Tools do software ArcGIS10.

\section{ANÁlises E FERRAMENTAS UTILIZADAS}

\subsection{Análise Espacial}

A análise espacial consiste na modelação da informação geográfica, sendo constituída por um conjunto de técnicas que tem por finalidade explicar o relacionamento espacial pre-sente num determinado fenómeno (CÂMARA et al., nd). Antes do cálculo de variação de densidade, é calcula a de densidade, através da técnica de kernel. Esta técnica calcula 
a magnitude por unidade de área (célula) usando a função de kernel (1), resultando numa superfície de informação "suave" e "contínua" (SILVERMAN, 1986).

$$
\hat{\int} \lambda(\chi)=\frac{1}{n \cdot \lambda} \cdot \sum_{i=1}^{\eta} \kappa_{0}\left(\frac{\chi-\chi i}{\lambda}\right)
$$

onde K0 é a função kernel e $\lambda$ é a largura de banda, n é o número total de features.

Existem várias funções que podem ser utilizadas para ponderar os valores de densidade (GIBIN et al., 2007). A mais usada é a função Epanechnikov (2) (SMITH et al., 2007):

$$
\frac{3}{4} \cdot\left(1-t^{2}\right) \text { para } t=\frac{d}{\lambda} \leq 1 \quad 0 \text { para } t=\frac{d}{\lambda}>1
$$

onde $d$ é a distância entre a célula e a feature e $\lambda$ é a largura da banda (raio de pesquisa).

\subsection{Estatística Espacial}

A estatística espacial reúne um conjunto de ferramentas capazes de descrever e modelar dados espaciais (SCOTT \& GETIS, 2008). De certa forma, desenvolvem o que a mente e os olhos fazem, intuitivamente, para avaliar padrões espaciais, distribuições, tendências, processos e relações (SCOTT \& JANIKAS, 2010; MITCHELL, 2005). A análise Hot Spot funci-ona num contexto de vizinhança e tem por base o cálculo estatístico $G i^{*}$ de Getis-Ord, que apresenta quais é que são os valores quentes (bot spot) ou frios (cold spot) através de um valor z-score (DIAS, 2013). Os cálculos são expressos da seguinte forma:

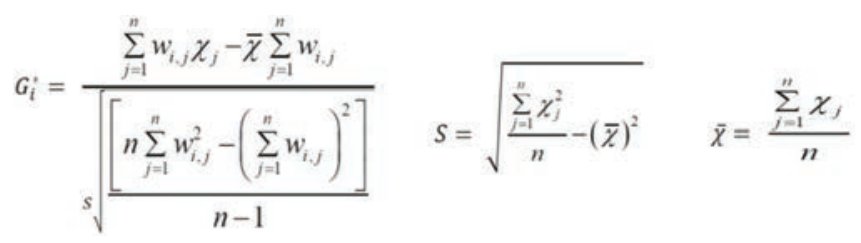

onde $X_{j}$ é o valor atributo da feature $j, W_{i, j}$ é o peso espacial entre a feature $i$ e $j, n$ é o número total de features. 
O kriging é uma técnica de interpolação que envolve uma estrutura de correlação espaci-al do fenómeno, de forma a calcular a interpolação estimativa e a variação de erro (SKEL-LY, 1993; DIAS, 2013), sendo expressa da seguinte forma:

$$
Z\left(\chi_{0}\right)=\sum_{i=0}^{n} \lambda_{i} Z\left(\chi_{i}\right)
$$

onde $\lambda i$ é o peso kriging determinado utilizando a função semivariâncias, $Z(X i)$ é o valor de uma feature de amostra $X i$, e $n$ é o número de features de amostragem.

\section{METODOLOGIA}

A informação baseia-se no o recenseamento comercial do retalho e restauração, dos anos 1995, 2000 e 2009. Nestes existe informação complementar, sendo esta o "número total de pessoal ao serviço" (TotP) e a "superfície de cliente" (SupC) / "superfície de exposição de venda" (SEV). A metodologia resume-se em três pontos: Variação de densidade do Reta-lho e da Restauração; Hot Spot Analysis do Retalho e Restauração do ano 2009; Kriging.

\section{Apresentação dos resultados}

No que diz respeito à variação da densidade da restauração (Figura 1) é de destacar um decréscimo muito alto no local da antiga Feira Popular de Lisboa (FPL); e acréscimos nos Centros Comerciais (C.C.) do Colombo e Vasco da Gama. Na variação da densidade da restauração SupC (Figura 2) é de notar o mesmo decréscimo na FPL e o acréscimo na mai-oria da cidade. Em relação à variação da densidade do retalho é de destacar o decréscimo geral na cidade, destacando a Baixa; acréscimos pontuais nos C.C. do Colombo e Vasco da Gama. No retalho SEV, para além do forte decréscimo, é de notar outras áreas de acrés-cimo, como o El Corte Inglês. Para a análise Hot Spot da restauração SupC (Figura 3) é de salientar a disposição de vários aglomerados de pontos "quentes" e "frios". Relativamen-te à análise Hot Spot da restauração TotP (Figura 
4) salienta-se a "expansão" da maioria das áreas com pontos "quentes" e "frios" analogamente ao anterior. Em relação à análise Hot Spot do retalho SEV, são apresentadas menos aglomerações de pontos "quentes" e "frios". Para a análise Hot Spot do retalho TotP verifica-se uma alteração significativa dos pontos frios no centro histórico e uma contracção dos aglomerados dos pontos "quen-tes". Através da análise Kriging (Figuras 5 e 6) é possível verificar as mesmas análises ex-traídas da análise de Hot Spots, com uma interpretação é mais "simples" dos resultados.

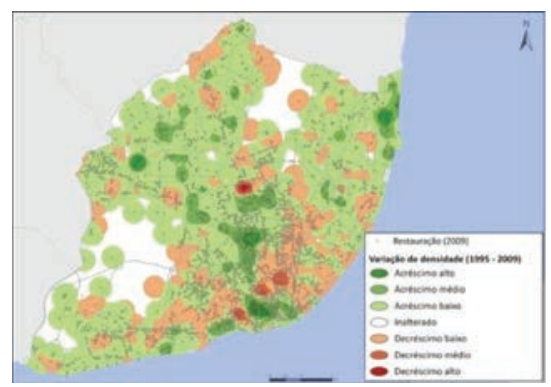

Figura 1 - Variação da Restauração (1995-2009)

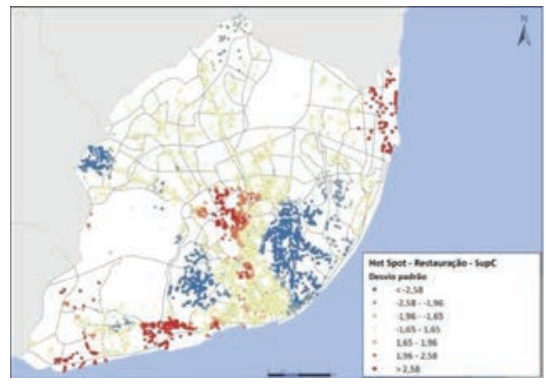

Figura 3 - Análise Hot Spot da Restauração TotP

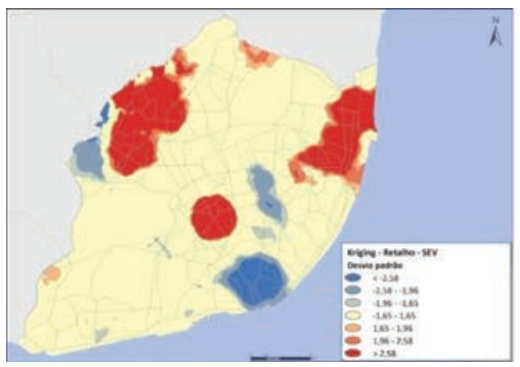

Figura 5 - Análise Kriging da Restauração SEV

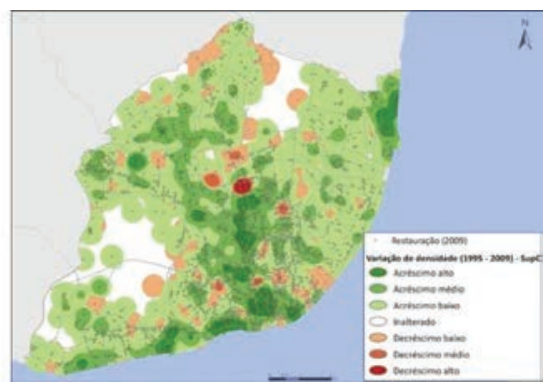

Figura 2 -Variação Restauração (1995-2009) SupC

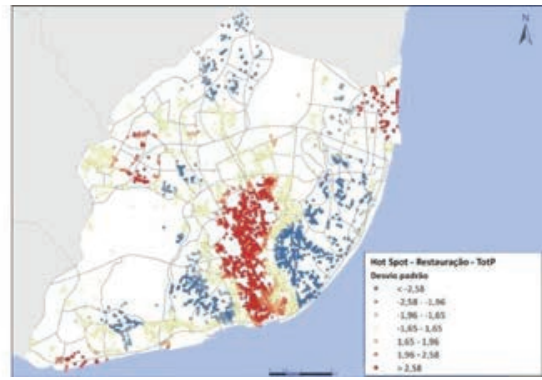

Figura 4 - Análise Hot Spot da Restauração SupC

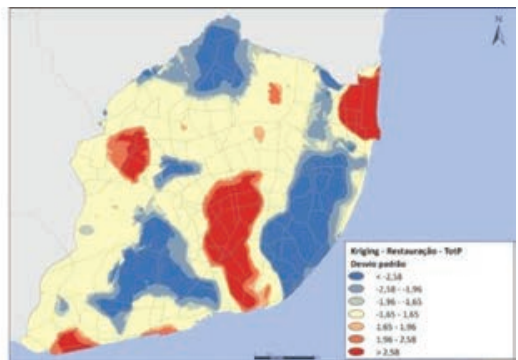

Figura 6 - Análise Kriging da Restauração TotP 


\section{CONSIDERAÇÕES FINAIS}

Todo este trabalho desenvolvido permitiu retractar qual ou quais realidades intrínsecas do sector estratégico do comércio, na cidade de Lisboa. Caso existisse mais informação complementar para os restantes sectores estratégicos, toda esta metodologia podia ser aplicada da mesma forma. O uso destas ferramentas permite obter este tipo de retrato, contudo a qualidade e quantidade de informação pré-existente é sempre um dos princi-pais critérios para que seja possível esta análise. Caso não seja, o resultado final será in-correcto e não representar a realidade.

\section{BIBLIOGRAFIA}

CÂMARA, Gilberto et al. (2004) - "Spatial Analysis and GIS: A Primer". Disponível online no endereço url: http://faculty.ksu.edu.sa/adosari/Documents/spatial_analysis_primer .pdf (acedido em 20 de Janeiro, 201;

DIAS, Pedro (2013) - "Análise exploratória de dados espaciais de actores de sectores es-tratégicos na cidade de Lisboa”, Relatório de Estágio de Mestrado, Lisboa: Faculdade de Ciências Sociais e Humanas, Universidade Nova de Lisboa;

GIBIN, Maurizio et al. (2007) - "Kernel Density Estimation and Percent Volume Contours in General Practice Catchment Area Analysis in Urban Areas”, in WINSTANLEY, Adam (ed.): Proceedings of the Geographical Information Science Research UK Conference 2007, County Kildare, Ireland. Disponível online no endereço url: http://www.geos.ed.ac.uk/ gis teac/proceedingsonline/GISRUK2007/PDF/5A3.pdf (acedido em 22 de Janeiro de 2014);

MITCHELL, Andy (2005) - "The ESRI Guide to GIS Analysis, Volume 2: Spatial Measure-ments and Statistics”, ESRI Press, Environmental Systems Research Institute, Redlands, California;

SCOTT, Lauren \& GETIS, Art (2008) - "Spatial statistics" in KEMP, Karen (ed.): Encyclopedia of geographic informations, Sage, Thousand Oaks, CA, 436-440. Disponível online no en-dereço url: http://www.springer.com/cda/content/ document/cda_downloaddocument/ 9783642036460-c1.pdf?SGWID=0-0-45855715-p173918949 (acedido a 8 de Abril, 2014);

SCOTT, Lauren \& JANIKAS, Mark (2010) - "Spatial Statistics in ArcGIS", in FISCHER, Manfred \& GETIS, Arthur (eds.): Handbook of Applied Spatial Analysis: Software Tools, Methods and Applications, Springer, Verlag Berlin Heidelberg, 27-41;

SILVERMAN, Bernard (1986) - "Density Estimation for Statistics and Data Analysis". In Monographs on Statistics and Applied Probability, London: Chapman 
and Hall. Disponível online no endereço url: http://ned.ipac.caltech.edu/ leve15/March02/Silverman/paper.pdf (acedido em 20 de Janeiro de 2014);

SKELLY, William (1993) - "Spatial interpolation: getting a realistic estimate of general cir-culation model precipitation”, in BOLLE, Hans-Jürgen, FEDDES, Reinder Auke \& KALMA, Jetse Daniel (eds.): Exchange Processes at the Land Surface for a Range of Space and Time Scales (Proc. IAHS/IAMAP/ UNESCO Symp., Yokohama, Jully 1993) IAHS Publ. no. 212, IAHS Press, Wallingford, UK, 603-610;

SMITH, Michael et al. (2007) - "Geospatial Analysis: A Comprehensive Guide to Principles, Techniques and Software Tools", Troubador Publishing. 
Série Documentos

Imprensa da Universidade de Coimbra

Coimbra University Press

2015

- U M

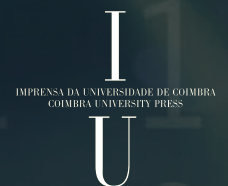

